
3 Research Square
Preprints are preliminary reports that have not undergone peer review.
They should not be considered conclusive, used to inform clinical practice,
or referenced by the media as validated information.

\title{
Thinking about the misdiagnosis: A case report of intra-abdominal desmoid- type fibromatosis and literature review
}

\author{
XuChang He \\ Kunming Medical University Second Hospital \\ Wenhui An \\ Kunming Medical University Second Hospital \\ Qingbin Zeng \\ Kunming Medical University Second Hospital \\ Kui Long \\ Kunming Medical University Second Hospital \\ Wenzhi Dong \\ Kunming Medical University Second Hospital \\ Xiaohua Mo ( 13708407263@139.com)
}

Case Report

Keywords: desmoid-type fibromatosis, soft tissue sarcoma, intra-abdominal type, misdiagnose, treatment, differential diagnosis, case report

Posted Date: March 13th, 2020

DOI: https://doi.org/10.21203/rs.3.rs-17084/v1

License: (c) (1) This work is licensed under a Creative Commons Attribution 4.0 International License. Read Full License 


\section{Abstract}

Background Desmoid-type fibromatosis is a low grade soft tissue sarcoma that derives from mesenchymal progenitor cells. Without characteristics of imaging, desmoid-type fibromatosis often confused with stromal tumors clinically. The disease characterized by local invasiveness and local recurrence but rare do metastasize. It can occur anywhere in the body. Most common location is the abdominal wall, nearly $30 \% \sim 50 \%$ in all cases. It can also occur in the abdominal cavity, with a low incidence. At present, all types of desmoid-type fibromatosis follow the treatment consensus of extra-abdominal type. However, because of the difficulty in preoperative diagnosis, sometimes it is hard to choose the appropriate treatment.

Case presentation We describe the case of a 22-years-old woman, who was diagnosed with an abdominal mass. She was a healthy individual who was incidentally diagnosed with abdominal mass. The mass was under the suspicion of gastrointestinal stromal tumors in a local hospital, according to the result of MRI scan. A CT scan of her abdomen showed a huge hypoechoic mass in the hepatic. Under misdiagnosis as hemangioma of liver in our hospital, she underwent a laparoscopic resection of the tumor. Postoperatively, the tumor was confirmed to be a desmoid-type fibromatosis by patholgical and immunohistochemical findings.

Conclusions In conclusion, due to the high risk of recurrence, the diagnosis before treatment is the most important part in the management of desmoid-type fibromatosis. Even if it is rare, DF should also be included in the differential diagnosis. Treatment needs to be evaluated according to the location of the lesion. For lesions on the body surface, pathologic examination will be safer and more useful. Surgery is still one of the best choice for intra-abdominal desmoid-type fibromatosis patients. Meanwhile, in order to change the operation in time, the clinicals should keep a rigorous attitude in surgery, if preoperative diagnosis is difficult.

\section{Introduction}

Desmoid-type fibromatosis,also known as aggressive fibromatosis or desmoid tumour. It is a rare, low grade soft tissue sarcoma that derives from mesenchymal progenitor cells. According to the Mayo Clinic, the annual incidence is between three and four parts per million ${ }^{[1]}$. Desmoid-type fibromatosis was first reported by MacFarlane in $1832 \square$ and it was named for the first time by Muller according to its general appearance and texture in $1838^{[2]}$. The disease characterized by local invasiveness and local recurrence, but rarely metastatic lesions ${ }^{[3]}$.The local recurrence rate is high ,between $15-77 \%{ }^{[4,5]}$.DF can occur in many places, the most common position are extremities and abdominal wall. It can also occur in abdomen al cavity, with a low incidence (15\% of DF cases). By the location of the disease, DF usually can be divided into three types: extra-abdominal, abdominal, or intra-abdominal. Due to the interference of abdominal visceral organs, intra-abdominal DF often confused with stromal tumors clinically. At present, all types of DF follow the treatment consensus of extra-abdominal type, which sometimes makes it hard to choose the appropriate treatment. Here, we present an uncommon case of one patient who was hard to diagnosis by imaging and a review of the relevant literature.

\section{Case Presentation}

A 22-years old women presented to the hospital with the complaint of an abdominal mass for a month. She was hospitalized for an abdominal mass in local hospital. Because there are no obvious symptoms, she had a good diet and sleep quality, with normal stools and urine, and no obvious change in weight. She was a healthy individual who had no specific medical history. She did not drink or smoke. The MRI scanning result showed a huge hypoechoic mass in her right abdominal cavity, measuring $12 \mathrm{~mm} \star 7 \mathrm{~mm}$. She was finally diagnosed with suspected stromal tumors in the local hospital.

On admission, the abdomen was soft, without varicosities, and a slight bulge could be seen in the right side. A mass with diameter of about $12 \mathrm{~cm}$ was palpable near the lower margin of the right costal arch. The mass was hard, with a smooth surface, a clear boundary, and poor mobility. Without rebound tenderness or guarding,abdominal MRI scanning showed a huge hypoechoic mass in the hepatic(Fig. 1). The mass had a clear boundary, smooth surface and an oval shape. The vascular enhancement was obvious at venous stage. Finally, the imaging diagnosis was considered as liver hemangioma. Due to the difference between the two MRI findings, the patient also assessed an abdominal CT scan, but the result was still the same as MRI. No GISTs was found by the gastroduodenoscopy results. In other laboratory examinations, there was no obvious abnormality, only slight increase of CA-125 in tumor markers. Medical history showed no abnormalities.

After evaluation was complete,the patient was recommended for a laparoscopic surgery. At surgery, the mass was a large white oval, compressing the liver. With a very tough surface and capsule, the mass seemed smooth. It was found suspected originated in the visceral peritoneum, which was suspended below the diaphragm. The mass was clearly squeezing the liver, but there was a boundary between them and there were no adhesions. The mass was completely excised from the diaphragm, and wound surface was carefully sutured to stop bleeding. To be on the safe side, except for complete resection of the tumor, it is worth mentioning that we set an incision protector while removing the tumor, according to the intraoperative diagnosis.

Pathological results showed a revealed DF of the visceral peritoneum (Fig. 2a,b ). Immunohistochemical findings were positive for nuclear beta-catenin, CD31, CD34, whereas SMA, ER, PR, Desmin, S100, CD117 were negative. There was less than $3 \%$ cells which were Ki67-positive. Because of the potential malignancy of the disease, the patient was required for returning to hospital every six months for follow-up. Up to now, there was no significant recurrence signs according to the imaging examination.

\section{Discussion And Conclusions Pathogeny}


The cause of the disease is still unclear. Some researchers showed that patients with familial adenomatous polyposis(FAP) have a higher risk of developing desmoid-type fibromatosis, comparing with non-FAP population ${ }^{[6]}$. Meanwhile, FAP related tumors are more likely to occur in the abdominal cavity ${ }^{[7]}$.0ther studies suggest that surgical trauma and neuroendocrinology may have an important role in the pathogenesis of $\mathrm{DF}^{[8,9]}$.Although the cause of $\mathrm{DF}$ remains controversy , most of them acknowledge that the pathogenesis should be a combination of multiple factors.

Mutations in the CTNNB1 gene, which encodes beta- catenin, are considered to be the key factor of pathogenesis in sporadic DF. CTTNB1 is located on chromsone 3p21 with 16 exons, which mainly encode beta-catenin. The mutation in the CTNNB1 gene that result in elevated beta-catenin appear to alter mesenchymal stem cell differentiation, and remarkably promoting the growth of tumour ${ }^{[1]}$. Beta-catenin is a transcriptional activator which significantly associated with a potential risk of recurrence ${ }^{[10]}$.In the case of CTTNB1 mutation, the incidence of DF can be significantly increased, from $39 \%$ to $87 \%$

${ }^{[11]}$.Therefore, mutation analysis of beta-catenin is regarded as a specific diagnostic tool for DF diagnosis.

\section{Diagnosis}

The preoperative diagnosis of desmoid-type fibromatosis is challenging. With no specificity on imaging, intra-abdominal DF is often confused with other soft tissue tumor, especially gastrointestinal stromal tumors (GISTs). Up to now, magnetic resonance imaging (MRI) is still the main means of imaging, which can be used for diagnosis, local staging and follow-up ${ }^{[12]}$.Although the key diagnostic feature in MRI has already pointed out for decades ${ }^{[13]}$,influenced by the experience of clinicians, the misdiagnosis rate is still high in the preoperative diagnosis of DF. Imaging is helpful to determine the location and invasion of DF, but it can hardly distinguish it from other soft tissue tumors. Meanwhile, due to the complexity of organizational structure, determining the origin of tumor by imaging means is also difficult. Hence, comparing with the diagnostic value, imaging may be more advantageous in management of the disease, especially when tumors occur in rare locations.

Pathologic examination is the primary means of diagnosis. However, nuclear accumulation of beta-catenin has been also observed in other soft tissue neoplasms. In this case, a mutations analysis of CTNNB1 should be performed『which is more specific to the diagnostic of DF ${ }^{[14]}$. Even DF is a rare disease, due to the high rate of recurrence, European Organization for Research and Treatment of Cancer (EORTC) suggested that needle biopsy should be mandatory prior before the treatment is started ${ }^{[15]}$. The accurate preoperative diagnosis of DF is still challenging, and a complete diagnostic strategy seems necessary. Recent research has been working toward this aspect. A study comes from Poland presented a short algorithm(Fig 3.) of immunostainings that can be useful in differential diagnosis ${ }^{[16]}$.

\section{Treatment}

The treatment of DF has changed in the past 10 years. Up to now. There is no standard treatment for DF. Therapeutic options include surgical resection, radiation therapy, chemotherapy, anti-inflammatory therapy, and hormone therapy.

Therapy is necessary when the disease causes obvious symptoms, if possible, surgical resection is still the first choice of treatment. According to the guide line of Nation Comprehensive Cancer Network (NCCN), surgical resection should be considered for patients with clinical symptoms or organ dysfunction ${ }^{[17]}$. But recently, there is a current shift to non-surgical approaches, named the "wait-and-see policy", due to the high risk of relapse after surgery. Even if it is still controversial, more and more investigators have recommended that the "wait-and-see policy" has been proposed and advocated as a viable approach to therapy ${ }^{[15]}$. This approach has been assessed by many different trails .A study from France comparing surgical versus "wait-and-see policy",which conducted a prospective study of 771 confirmed cases, found that these was no significant difference in prognosis between the two groups . The studies also pointed out that the position of DF can affect prognosis, especially in the abdominal wall, abdominal cavity ,breast, digestive organ and lower limbs ${ }^{[18]}$. $\mathrm{A}$ meta-analysis with a large sample size yielded similar results ${ }^{[19]}$. Therefore, compared with other disease, the surgical treatment of DF should be chosen with caution, a personalized treatment strategies are necessary according to the changing consensus ${ }^{[20]}$.

Radiation and systemic therapy are recommended for patients with unresectable or recurrent disease. Some researchers suggested that radiation therapy cannot reduce the risk of local recurrence after surgery, especially for patients with an initial negative margin ${ }^{[21]}$. Meanwhile, other researchers had found positive effects for those who suffered multiple operations or progressive disease ${ }^{[22]}$. Thus, radiation therapy is regarded as an adjuvant therapy for incomplete resection or recurrence. For such patients, a systemic therapy also could be helpful. While there are many options, including chemotherapy, hormone therapy, target therapy and anti-inflammatory therapy, the therapeutic effect are generally poor. Further research of drugs are still needed.

\section{Comment}

According to the above consensus, there were some different that should be pointed out in our treatment process. Although it is recommended for both NCCN and EORTC, we did not have a needle biopsy before the surgery. Why? Our MRI scan incorrectly revealed a complex mass in the liver, which could be a stromal tumor, a hemangioma or something else. In this case, a needle biopsy may cause significant bleeding, breaking or metastasis. At the same time, these complications will be more danger while occurred in the abdominal cavity. On the other sides, making a definitive diagnosis for using a fine needle aspirate sometimes seems to be difficult, especially for the intra-abdominal mass. Regardless of the result, in this case, it may lead to obvious disputes due to different cultures in the East and the West. Different from DF in other position, in case of no accurate pathological diagnosis before operation, a "wait-and-see policy" seems be not appropriate. In that way, for Chinese patients with intra-abdominal DF, the treatment seems to be contradictory when following the consensus. On the other hand, it may also cause more risks for clinicians. 
Intra-abdominal DF is often confused with gastrointestinal stromal tumors, meanwhile, the two diseases are similar in tumor behavior. Besides, some researchers suggested that DF may be controlled by the same genes in the process of the disease with GISTs ${ }^{[23,24]}$. But what

$s$ ferest $\in$ gist $\hat{t} h e \in$ cnce and treatmentcasesofGISTsishigherthanDF. Thus, $f$ or thisraredisease, isitafeasib $\leq$ method $\rightarrow$ trea s guideline of GISTs, surgery treatment is the first choice treatment ${ }^{[25]}$ ). There is some moderate evidence suggest surgery may be more helpful for those patients who are suitable for complete resection, considering of difficult preoperative diagnosis ${ }^{[26]}$. The association of two disease may need further studies to elucidate. Besides, a complete surgical exploration before remove it for this rare disease is always necessary. The clinicals should keep a rigorous attitude, especially when preoperative diagnosis is difficult. In the case of misdiagnosis, this maybe the reason why the operation is effective for this patient. Also, for further understanding of DF, we have a simple summary of the three kinds of diagnosis in this case (Table.1).

The treatment consensus of DF is very meaningful, for lesions on the body surface, pathologic examination will be safer and more useful. But whether the intra-abdominal DF should be treated as other type of DF (especially for extra-abdominal DF) needs further study.

Table 1

Briefly distinguishing features of DF/GISTs/Liver hemangioma

\begin{tabular}{|c|c|c|c|}
\hline & DF & GISTs & Liver hemangioma \\
\hline \multicolumn{4}{|l|}{ Epidemiology } \\
\hline Morbidity & 2-3 per Million & 11-14.5 per Million & 0.4-7.3 Percent \\
\hline Age peak & No characteristic & $40-60$ & $40-60$ \\
\hline Gander & $\begin{array}{l}\bigotimes 40, M=F \\
\varangle 40, M \square F\end{array}$ & $M=F$ & $\mathrm{M} \square \mathrm{F}$ \\
\hline Location & Arm $\square$ Abdominal wall & Stomach,small intestine & Liver \\
\hline Origin & Interstitial cells of Cajal & mesenchymal progenitor cells & Vascular dysplasia of embryo \\
\hline Pathogenesis & $\begin{array}{l}\text { Unclear, maybe related to the mutation of } \\
\text { CTNNB1 gene }\end{array}$ & Most of them are c-kit mutations & $\begin{array}{l}\text { Unclear, maybe related to congenital } \\
\text { angiodysplasia }\end{array}$ \\
\hline $\begin{array}{l}\text { Immunological } \\
\text { marker }\end{array}$ & $\beta$-catenin nuclear gathering & CD1174DoG-1 expression & No \\
\hline \multicolumn{4}{|l|}{ Tumor behavior } \\
\hline $\begin{array}{l}\text { Distant } \\
\text { metastasis }\end{array}$ & No & No & No \\
\hline Partial invasion & Yes & Yes & No \\
\hline Local recurrence & Yes & Yes & No \\
\hline \multicolumn{4}{|l|}{$\begin{array}{l}\text { Tumor } \\
\text { configuration }\end{array}$} \\
\hline Color & White & White & Red \\
\hline Texture & Hard & Hard & Soft \\
\hline Capsule & Most of them no & Most of them no & Yes \\
\hline Symptom & No obvious symptoms & No obvious symptoms & No obvious symptoms \\
\hline Diagnosis & $\begin{array}{l}\text { Pathological examination,no } \\
\text { characteristics of imaging }\end{array}$ & $\begin{array}{l}\text { Pathological examination,no } \\
\text { characteristics of imaging }\end{array}$ & Imaging examination \\
\hline Treatment & Wait and see,Surgery (still controversial) & Surgery & Surgery \\
\hline
\end{tabular}

\section{Conclusion}

In conclusion, due to the high risk of recurrence, the diagnosis before treatment is the most important part in the management of DF. Even if it is rare, DF should also be included in the differential diagnosis. Treatment needs to be evaluated according to the location of the lesion. For lesions on the body surface, pathologic examination will be safer and more useful. If preoperative diagnosis is difficult, surgery is still one of the best choice for intra-abdominal DF patients. Meanwhile, in order to change the operation in time, the clinicals should keep a rigorous attitude in surgery.

\section{Abbreviations}

DF: Desmoid-type fibromatosis; MRI: Magnetic Resonance Imaging; CT: Computed Tomography; FAP: Familial adenomatous polyposis; GISTs: Gastrointestinal stromal tumors; EORTC: European Organization for Research and Treatment of Cancer; NCCN: Nation Comprehensive Cancer Network;

\section{Declarations}


Not applicable.

Authors contributions

Mr. He and Ms. An contributed equally to this work. Pro. Mo, Pro. Long, Mr. Zeng and Mr. Dong performed the clinical treatment. All authors contributed to the writing and revision of the manuscript.

Funding

Not applicable.

Availability of data and materials

The datasets used and/or analyzed during the current study are available from the corresponding author upon reasonable request.

\section{Ethics approval and consent to participate}

The study protocol was approved by the Second Affiliated Hospital of Kunming Medical University and written informed consent was obtained from the patient.

\section{Consent for publication}

Informed consent for publication was obtained from the patient.

\section{Competing interests}

The authors declare that they have no conflicts of interest.

\section{Author details}

Department of Hepatobiliary Surgery. The Second Affiliated Hospital of Kunming Medical University. Kunming, 650000, P.R. China

\section{References}

1. Skubitz, M. K. Biology and Treatment of Aggressive Fibromatosis or Desmoid Tumor[J]. Mayo Clinic Proceedings 2017; 92(6):947-964.

2. Mullen JT, DeLaney TF, Rosenberg AE, et al. î-Catenin Mutation Status and Outcomes in Sporadic Desmoid Tumors[J]. Oncologist 2013; 18 (9):1043-1049.

3. Penel N, Chibon F, Salas S. Adult desmoid tumors: biology, management and ongoing trials[J]. Current Opinion in Oncology $2017 ; 29$.

4. Ballo MT, Zagars GK, Pollack A, et al. Desmoid Tumor: Prognostic Factors and Outcome After Surgery, Radiation Therapy, or Combined Surgery and Radiation Therapy[J]. Journal of Clinical Oncology 1999; 17(1):158-158.

5. S?rensen A, Keller J, Nielsen OS, et al. Treatment of aggressive fibromatosis: A retrospective study of 72 patients followed for 1-27 years[J]. Acta Orthopaedica 2002.

6. Nieuwenhuis MH, Casparie M, Mathus-Vliegen LM, et al. A nation-wide study comparing sporadic and familial adenomatous polyposis-related desmoidtype fibrom atoses[J]. Int J Cancer 2011; 129(1):256-261.

7. L K, A R, A L. Comparison of sporadic and FAP-associated desmoid-type fibromatoses[J]. Journal of Surgical Oncology 2017; 116(6).

8. Siaudinyte I, Spiliauskaite S, Arlauskiene A, et al. Rapid Growth of a Giant Intra-Abdominal Desmoid Tumor Diagnosed During Pregnancy[J]. The American Surgeon 2019; 85(4):e204-e207.

9. Dumont AG, Rink L, Godwin AK, et al. A nonrandom association of gastrointestinal stromal tumor (GIST) and desmoid tumor (deep fibromatosis): case series of 28 patients[J]. Annals of Oncology 2012; 23(5):1335-1340.

10. Kim JS, Kim HJ, Lee MY, et al. Survival outcomes after adjuvant radiotherapy for aggressive fibromatosis depend on time frame and nu clear |Âcatenin[J]. Radiat Oncol J 2019; 37(1):37-42. 
11. Lazar AJF, Tuvin D, Hajibashi S, et al. Specific Mutations in the $\beta$-Catenin Gene (CTNNB1) Correlate with Local Recurrence in Sporadic Desmoid Tumors[J]. American Journal of Pathology 2008; 173(5):0-1527.

12. Benson C, Miah AB, Otero S, et al. Desmoid-type fibromatosis[J]. Clinical Radiology: Journal of the Royal College of Radiologists 2015; 70(9):1038-1045.

13. Healy JC, Reznek RH, Clark SK, et al. MR appearances of desmoid tumors in familial adenomatous polyposis[J]. American Journal of Roentgenology 1997; 169(2):465-472.

14. Le Guellec S, Soubeyran I, Rochaix P, et al. CTNNB1 mutation analysis is a useful tool for the diagnosis of desmoid tumors: a study of 260 desmoid tumors and 191 potential morphologic mimics[J]. Modern Pathology 2012; 25(12):1551-1558.

15. Kasper B, Baumgarten C, Garcia J, et al. An Update On the Management of Sporadic Desmoid-Type Fibromatosis: A European Consensus Initiative Between Sarcoma Patients Euronet (Spaen) And European Organisation For Research and Treatment of Cancer (Eortc)/Soft Tissue and Bone Sarcoma Group (STBSG)[J]. Annals of Oncology 2017.

16. Lacka DE, Nasierowska-Guttmejer A. Fibromatosis - immunohistochemical evaluation, differential diagnosis from gastrointestinal tumors, a nd other mesenchymal tumours[J]. Prz Gastroentero/ 2019; 14(1):79-85.

17. Mehren Mv, Randall RL, Benjamin RS, et al. Soft Tissue Sarcoma, Version 2.2018, NCCN Clinical Practice Guidelines in Oncology[J]. Journal of the National Comprehensive Cancer Network: JNCCN 2018; 16(5):536-563.

18. Penel N, Le Cesne A, Bonvalot S, et al. Surgical versus non-surgical approach in primary desmoid-type fibromatosis patients: A nationwide pro spective cohort from the French Sarcoma Group[J]. Eur J Cancer 2017; 83:125-131.

19. Seinen JM, Niebling MG, Bastiaannet E, et al. Four different treatment strategies in aggressive fibromatosis: A systematic review[J]. Clinical and Translational Radiation Oncology 2018:S2405630818300041.

20. Howard JH, Pollock RE. Intra-Abdominal and Abdominal Wall Desmoid Fibromatosis[J]. Oncology and Therapy 2016; 4(1):57-72.

21. Mastoraki A, Schizas D, Vergadis $C$, et al. Recurrent aggressive mesenteric desmoid tumor successfully treated with sorafenib: A case report and literature review[J]. World J Clin Oncol 2019; 10(4):183-191.

22. Hee CS, In YH, Hyun KS, et al. Optimal radiotherapy strategy for primary or recurrent fibromatosis and long-term results[J]. PLOS ONE 2018; 13(5):e0198134-

23. Ihalainen HR, Koljonen V, B?hling TO, et al. The desmoid tumour: Local control after surgical treatment[J]. Journal of Plastic Surgery \& Hand Surgery 2015; 49(1):19-24.

24. Dumont AG, Rink L, Godwin AK, et al. A nonrandom association of gastrointestinal stromal tumor (GIST) and desmoid tumor (deep fibromatosis): case series of 28 patients[J]. Annals of Oncology Official Journal of the European Society for Medical Oncology 2012; 23(5):1335-1340.

25. Demetri GD, von Mehren M, Antonescu CR, et al. NCCN Task Force report: update on the management of patients with gastrointestinal stromal tumors[J]. J Natl Compr Canc Netw 2010; 8 Suppl 2:S1-41; quiz S42-44.

26. Wang Z, Wu J, Lv A, et al. En bloc resection for intra-abdominal/retroperitoneal desmoid-type fibromatosis with adjacent organ i nvolvement: A case series and literature review[J]. Biosci Trends 2018; 12(6):620-626.

\section{Figures}



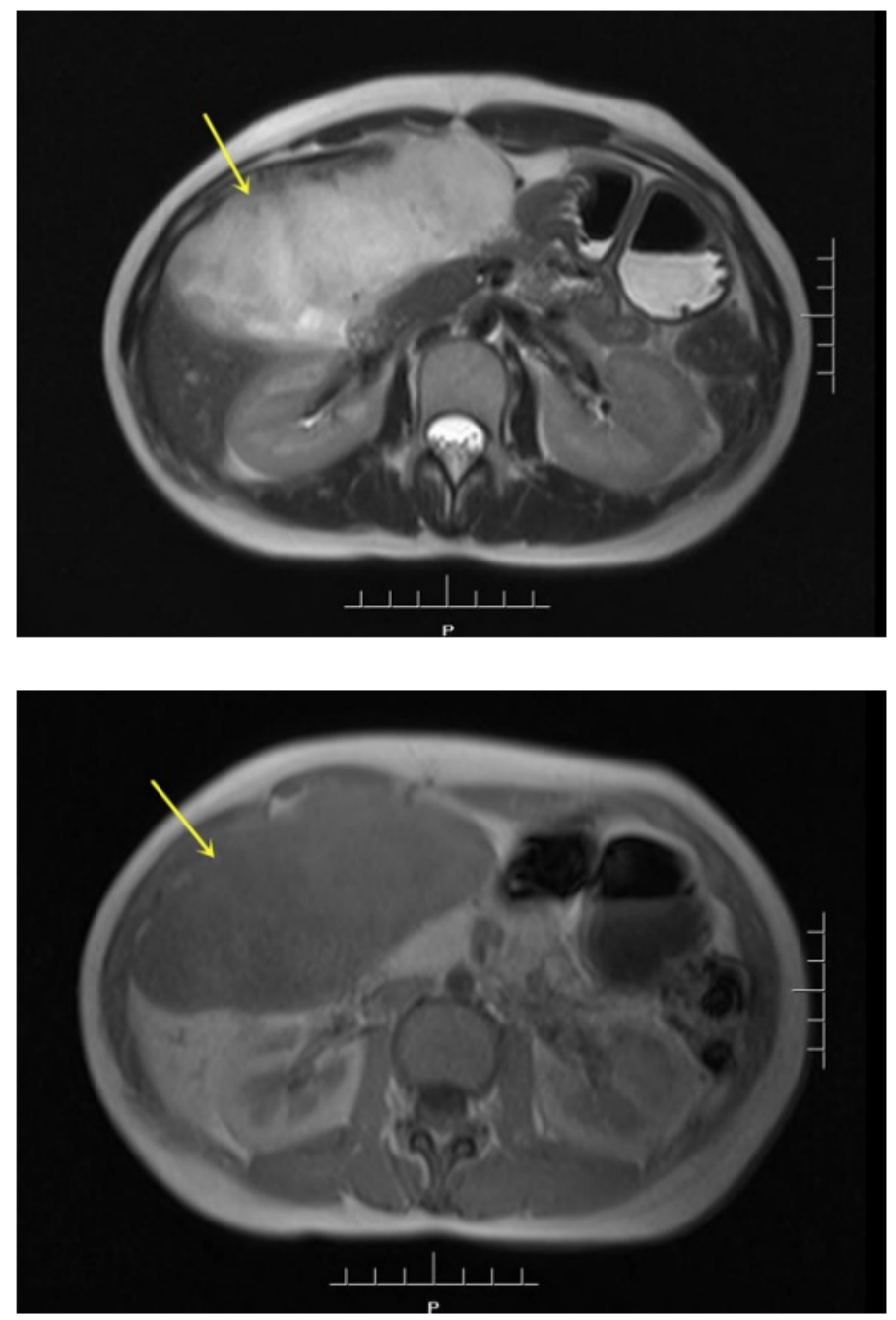

\section{Figure 1}

The abdominal MRI scanning showed a huge hypoechoic mass in the hepatic, with long T1 and T2 signal , measuring $13.35 \mathrm{~cm} * 13.15 \mathrm{~cm} * 6.32 \mathrm{~cm}$. The vascular enhancement was obvious at venous stage. 

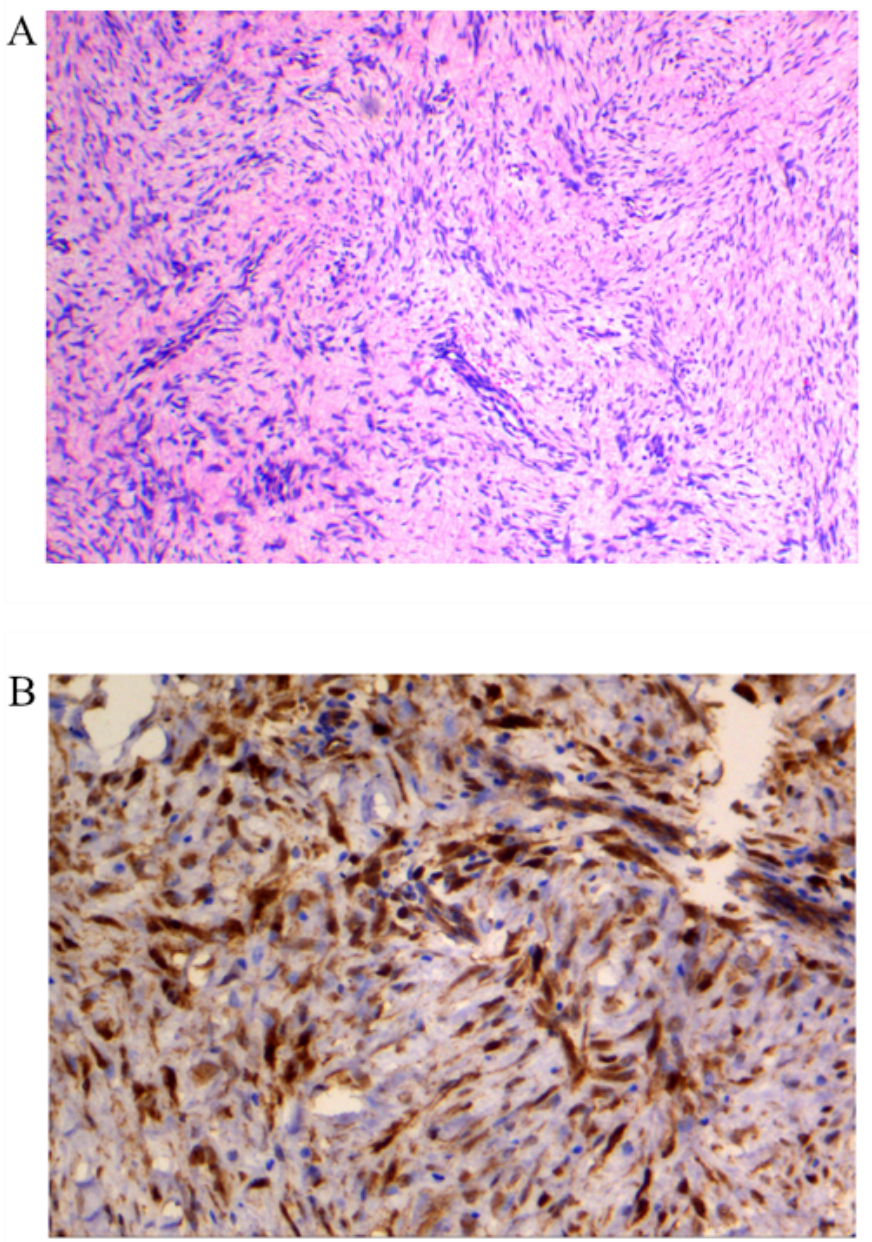

\section{Figure 2}

A. details showing low-grade fusocellular proliferation and delicately fibrillated cytoplasm (magnification $\times 100$, hematoxylin/eosin). B. strongly positive for beta-catenin (magnification $\times 200$ ) . 


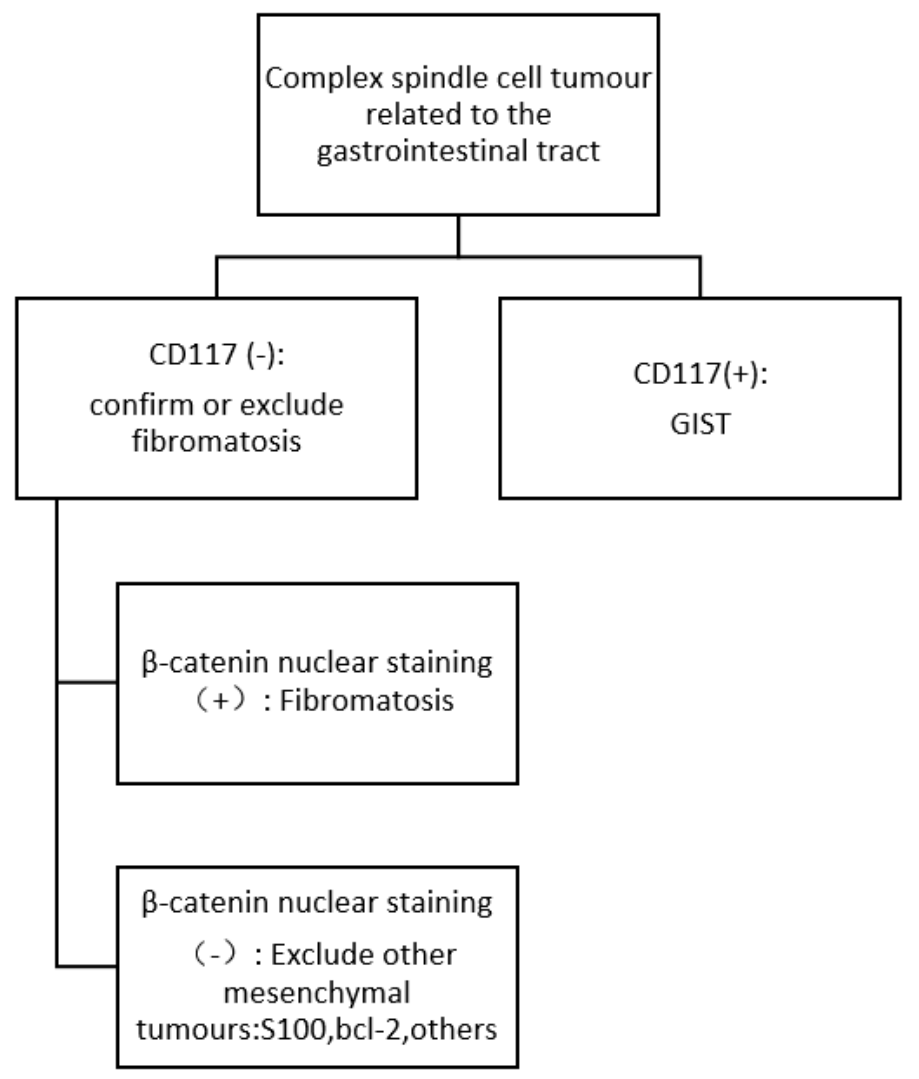

Figure 3

Short diagnostic strategy in differential diagnosis of complex fibromatosis. 